\title{
Production of extracts with anaesthetic activity from the culture of Heterosigma akashiwo in pilot-scale photobioreactors
}

\author{
J.J. Gallardo-Rodríguez ${ }^{\mathrm{a}, \mathrm{b}, *}$, A. Astuya-Villalón ${ }^{\mathrm{c}}, \mathrm{V}$. Avello ${ }^{\mathrm{c}}$, A. Llanos-Rivera ${ }^{\mathrm{c}}, \mathrm{B}$. Krock ${ }^{\mathrm{d}}$, \\ C. Agurto-Muñoz ${ }^{\mathrm{e}, \mathrm{f}}$, A. Sánchez-Mirón ${ }^{\mathrm{a}}, \mathrm{F}$. García-Camacho ${ }^{\mathrm{a}}$ \\ ${ }^{a}$ Department of Chemical Engineering, University of Almería, Spain \\ ${ }^{\mathrm{b}}$ Department of Chemical Engineering, Faculty of Engineering, University of Concepción, Concepción, Chile \\ ${ }^{\mathrm{c}}$ Laboratory of Cell Culture and Marine Genomics, Department of Oceanography, Faculty of Natural and Oceanographic Sciences, COPAS Sur-Austral Program, University \\ of Concepción, Concepción, Chile \\ ${ }^{\mathrm{d}}$ Alfred Wegener Institut-Helmholtz Zentrum für Polar- und Meeresforschung, Chemische Ökologie, Am Handelshafen 12, 27570 Bremerhaven, Germany \\ ${ }^{\mathrm{e}}$ Department of Food Science and Technology, Faculty of Pharmacy, University of Concepcion, Concepcion, Chile \\ ${ }^{\mathrm{f}}$ GIBMAR, Biotechnology Centre, Concepción University, Concepción, Chile
}

\section{A R T I C L E I N F O}

\section{Keywords:}

Raphidophytes

Anaesthetic

Bioprocess

Photobioreactor

\begin{abstract}
A B S T R A C T
The shear-sensitive microalga Heterosigma akashiwo is known to produce brevetoxin-like compounds that are active in voltage-dependent sodium channels. In this work, we present a study on the production of anaesthetic extracts from $H$. akashiwo biomass obtained in low-shear bioreactors at different growth phases. The photobioreactors (PBRs) used had specific configurations and were operated in such a way as to avoid cellular damage by hydrodynamic stress. Cultures were developed in small static-control flasks and PBRs with volumes ranging from $1.5 \mathrm{~L}$ to $200 \mathrm{~L}$. The bioactivity of the produced extracts was assessed in vitro (Neuro-2a cell-based assay) and in vivo (Zebra fish model). Bioactivity depended slightly on the growth phase and culture system, with greater toxicity with the Neuro-2a model when stationary-phase extracts were used. Interestingly, extracts were not cytotoxic against other human cell lines. Steady production of anaesthetic bioactives was observed. In vivo anaesthetic efficacy, assessed with the Zebra fish model, was similar to that of commercial products, and without any observed mortality at 24-h post exposure.
\end{abstract}

\section{Introduction}

Dinoflagellate and raphidophyte microalgae have gained biotechnological importance in recent years due to their ability to produce bioactive molecules, mostly polyketides. The wide inter- and intraspecies diversity implies the potential of discovering new molecules [1]. However, because it is difficult to obtain stable and repeatable cultures of these microalgae species, there have been only a few marketable applications to date [2]. Polyketides from fungi and microalgae are among the chemical groups screened for antitumor drugs. Other interesting bioactivities of this family of structures that are being studied as potential therapeutic drugs include immunosuppressors and drugs for infectious diseases [3]. Most dinoflagellate and raphidophyte microalgae produce multiple bioactive compounds, some of which are chemical species that typically share structural features (e.g. polyketides) but have different bioactivities. For instance, Karenia brevis synthesises brevetoxins and its antagonist, brevenal [4]. While this is interesting from an ecological or chemical point of view, it is challenging for bioprocessing. In these cases, culture conditions that trigger the synthesis of bioactives must be determined. The bioreactor culture continues to be the only alternative for obtaining microalgal bioactives. Although technologically possible and an option for derivatives, chemical synthesis is not feasible from an economic point of view.

Lack of knowledge about metabolism and optimal growth conditions and conditions to enhance bioactive production are among the difficulties in culturing dinoflagellate and raphidophyte microalgae [2]. With Protoceratium reticulatum, it has been observed that certain operating conditions increase toxin cell content, such as the level of agitation [5]. However, stable long-term agitated cultures significantly decrease yessotoxin production [6]. The major culture challenge is microalgal sensitivity to hydrodynamic stress and bioreactor design. Concentrations of dinoflagellate microalgae in photosynthetic cultures of dinoflagellate have generally been low compared to those of nondinoflagellate microalgae, although there have been advances in recent

\footnotetext{
* Corresponding author at: Department of Chemical Engineering, University of Almería, Carretera Sacramento s/n, E04120 Almería, Spain.

E-mail address: jgr285@ual.es (J.J. Gallardo-Rodríguez).
} 
years. Recent works with Amphidinium and Karlodinium have demonstrated that bioprocesses are feasible with some shear-sensitive species $[7,8]$. In highly controlled systems, biomass concentrations can reach nearly $1 \mathrm{~g} \cdot \mathrm{L}^{-1}$ [7]. Nevertheless, metabolite production is still a bottleneck because of the low quantities (pg per cell) and the high degree of dependence on culture conditions [6,9].

Raphidophytes like Heterosigma akashiwo, Fibrocapsa japonica, Chattonella spp. and Pseudochattonella are known to cause mass mortalities of cultivated and wild fish [10]. In particular, H. akashiwo has had an enormous impact on the coasts of Japan [11] and in other parts of the world $[12,13]$. Attempts have been made to determine the ichthyotoxic mechanisms of most HAB-forming species [14]. Proposed mechanisms include the production of reactive oxygen species (ROS) as the main source of damage, although the release of high free fatty acids has a synergistic effect. There is also an undeniable ichthyotoxic effect of toxins produced by several species, including H. akashiwo [15-17]. Some of these molecules can cause respiratory and/or cardiac paralysis and abnormal behavior $[17,18]$. The raphidophyceae class (e.g. $H$. akashiwo) has been suggested as a brevetoxin or brevetoxin-like compound producer [19], although some authors have questioned this [20]. The amount of brevetoxin and brevetoxin-like compounds produced is highly variable, and can even be at undetectable levels [19-21]. The crude extract of $H$. akashiwo CCMP302 showed an inhibitory effect on the voltage-dependent sodium channel in the neuronal model neuro-2a [16]. Interestingly, this suggests the presence of compounds with anaesthetic activity, with potential commercial value because their effects are similar to those of saxitoxin and tetrodotoxin [16].

This work reports on the feasibility of the microalga $H$. akashiwo CCMP302 to produce valuable bioactives when it is cultured photoautotrophically at different scales. Biomass extracts harvested at different growth phases were evaluated for anaesthetic activity (models based on Zebra fish and Neuro-2a CBA) and cytotoxicity.

\section{Materials and methods}

\subsection{The microalga}

The marine microalga Heterosigma akashiwo (strain CCMP302) was used. The alga was obtained from the Provasoli-Guillard National Center for the Culture of Marine Phytoplankton (Maine, USA). Inocula of $H$. akashiwo were grown in static flasks (without hydrodynamic stress) at $20 \pm 1{ }^{\circ} \mathrm{C}$ under continuous illumination. $58 \mathrm{~W}$ fluorescent lamps were used for illumination and the irradiance at the surface of the culture flasks was $100 \mu \mathrm{mol}$ photon $\mathrm{m}^{-2} \mathrm{~s}^{-1}$. Flasks were not aerated, but gently agitated manually once a day. The culture medium was supplemented with L1 and prepared in autoclaved natural seawater [22].

\subsection{Cultivation in photobioreactors}

Two types of PBRs were used for microalgal biomass production: (i) a 1.5-L conventional stirred glass bioreactor (New Brunswick, BioFlo model No. 030-032) with a six-bladed Rushton turbine as an impeller (SPBR1.5); (ii) three bubble columns with culture capacities of $2 \mathrm{~L}$ (PBR2), 12 L (PBR12) and 200 L (PBR200). The SPBR1.5 was baffled and had the following relevant dimensions: $0.11 \mathrm{~m}$ culture depth; $0.11 \mathrm{~m}$ vessel diameter; $1.5 \mathrm{~cm}$ clearance of the impeller from the bottom of the vessel; $0.05 \mathrm{~m}$ impeller diameter. The impeller blades were $0.75 \mathrm{~cm}$ long and $0.4 \mathrm{~cm}$ wide.

The three bubble columns, PBR2, PBR12 and PBR200, were tubes made of transparent polyvinyl chloride (PVC; Type I, Grade I compound with a cell classification of 12,454 per ASTM D1784). The tubes had internal diameters of $0.11 \mathrm{~m}, 0.14 \mathrm{~m}$ and $0.45 \mathrm{~cm}$, respectively. Cell suspensions were agitated by air sparging, with filtered air from a compressor and injected through a nozzle sparger placed at the bottom of the column. The gas-free liquid heights were approximately $0.78 \mathrm{~m}$,
$1.25 \mathrm{~m}$ and $1.25 \mathrm{~m}$, respectively.

Photoautotrophic growth of $H$. akashiwo was investigated in the PBRs. The culture temperature was controlled at $20 \pm 1{ }^{\circ} \mathrm{C}$. The culture $\mathrm{pH}$ was not controlled. In all cases the initial $\mathrm{pH}$ was set at 8.1. All PBRs were continuously illuminated with spirally wound cool-whitelight LEDs. The irradiance at the center of the photobioreactor (filled with seawater) was measured using a QSL-100 quantum scalar irradiance sensor (Biospherical Instruments, San Diego, USA). Incident irradiance (photosynthetically active) was $100 \mu \mathrm{mol}$ photon $\mathrm{m}^{-2} \mathrm{~s}^{-1}$. The photobioreactors and associated pipework were cleaned before the experiments to remove salt deposits and sterilized as described previously [6]. The culture medium was prepared as described above. The fresh medium was inoculated with $10 \% \mathrm{v} / \mathrm{v}$ of an inoculum containing algal cells in the late exponential growth phase. The cell concentration in the freshly inoculated photobioreactors was around 20,000 cells $\mathrm{mL}^{-1}$.

Two experimental sets were carried out. Firstly, as a prior step to PBR mass culture, tolerance of $H$. akashiwo to averaged energy dissipation rates (EDRs) developed in mechanical (SPBR1.5) and pneumatic photobioreactors (PBR2) was characterized with short-term cultures $(250 \mathrm{~min})$. The agitation rate $(N)$ was varied from $50 \mathrm{rpm}$ to $200 \mathrm{rpm}$ in SPBR1.5. The specific air flow rate $(Q)$ was varied from 0.2 to $0.6 \mathrm{v} \mathrm{v}^{-1} \mathrm{~min}^{-1}$ in PBR2. The assays are summarized in detail in Table 1. Once EDR-damage thresholds were determined from the results of the first experimental set. A conservative $Q$ value was selected to mass culture $H$. akashiwo in the aerated photobioreactors PBR12 and PBR200. The effect of scaling-up was also studied in the second set. A culture in a 1-L flask was used as a control. The cultures were produced batchwise in all PBRs.

\subsection{Hydrodynamic characterization of photobioreactors}

The acid-tracer method as described previously was used to measure the mixing time $\left(\theta_{m}\right)$ [6]. Briefly, the $\mathrm{pH}$ level was altered by adding hydrochloric acid (35\% wt/vol). The mixing time was calculated as the time elapsed to reach $95 \%$ of the final $\mathrm{pH}$ value. All experiments were carried out in triplicate. The $E D R$ value in the stirred bioreactor was calculated using the broadly applicable method of Ruszkowski [23], as follows:

$E D R=\left(\frac{A \cdot T}{\theta_{m} \cdot D^{1 / 3}}\right)^{3}$

where $A$ is a constant with a value of $5.9, D$ is the impeller diameter (m), $T$ is the tank diameter (m) and $\theta_{m}$ is the measured mixing time. Eq. (1) indicates that the relationship between $E D R$ and $\theta_{m}$ is independent of the type of stirrer. $E D R$ in the bubble columns is related to surface gas velocity $\left(u_{g}\right)$, according to Eq. (2) [24],

$E D R=g \cdot u_{g}$

where $\mathrm{g}$ is gravitational acceleration constant. The $u_{g}$ value can easily

Table 1

Summary of the experimental design to investigate the tolerance of $H$. akashiwo to the hydrodynamic stress in mechanically agitated (SPBR1.5) and aerated (PBR2) cultures. (N: impeller rotational speed; EDR: averaged energy dissipation rate; $\theta_{m}$ : mixing time; $\lambda$ : Kolmogorov's microscale of turbulence).

\begin{tabular}{lllrrl}
\hline PBR & $N, \mathrm{rpm}$ & $Q, \mathrm{v} \mathrm{v}^{-1} \mathrm{~min}^{-1}$ & $\theta_{m}, \mathrm{~s}$ & $E D R, \mathrm{~mW} \mathrm{~kg}^{-1}$ & $\lambda, \mu \mathrm{m}$ \\
\hline \multirow{2}{*}{ SPBR1.5 } & 50 & - & 14.0 & 1.8 & 51.6 \\
& 100 & - & 9.3 & 6.9 & 36.9 \\
& 150 & - & 6.5 & 19.9 & 28.4 \\
& 200 & - & 6.0 & 25.3 & 26.7 \\
PBR2 & 250 & - & 5.5 & 32.9 & 25.0 \\
& - & 0.2 & 16.0 & 3.4 & 44.0 \\
& - & 0.4 & 10.5 & 6.9 & 37.0 \\
& - & 0.6 & 6.3 & 10.3 & 33.4 \\
\hline
\end{tabular}


be obtained by dividing the gas flow rate by the column cross-section area. Kolmogorov's microscale of turbulence was obtained from the following equation:

$\lambda=\left(\frac{\mu_{L}}{\rho_{L}}\right)^{3 / 4} \cdot E D R^{-1 / 4}$

where $\mu_{L}$ and $\rho_{L}$ are broth viscosity and density, respectively. Table 1 shows the values of $\theta_{m}, E D R$ and $\lambda$ for the first experimental set.

\subsection{Kinetic parameters}

Growth was determined by cell counts. Broth samples $(1 \mathrm{~mL})$ were collected daily, fixed with Lugol's solution, and then cells were counted on a Sedgewick-Rafter counting slide. The maximum cell-specific growth rate $\left(\mathrm{h}^{-1}\right)$ in the exponential growth phase was calculated using the following equation:

$\mu_{\max }=\frac{\ln \left(N / N_{0}\right)}{t-t_{0}}$

where $N$ and $N_{0}$ are cell concentrations (cells $\mathrm{mL}^{-1}$ ) at culture times $t$ and $t_{\mathrm{o}}$ (days), respectively, in the interval when the specific growth rate is at a maximum. The doubling time $\left(T_{\mathrm{d}}\right)$ was calculated as:

$T_{d}=\frac{\operatorname{Ln}(2)}{\mu_{\max }}$

The maximum cell-specific death rate $k_{\mathrm{d}}\left(\mathrm{h}^{-1}\right)$ in short-term culture experiments to determine EDR thresholds was calculated using the following equation:

$\ln \left(N / N_{0}\right)=-k_{d} \bullet t$

where $N$ and $N_{O}$ are the cell concentrations (cells $\mathrm{mL}^{-1}$ ) at times $t$ and initial time, respectively, and $k_{d}$ is the maximum death rate.

\subsection{Preparation of H. akashiwo extracts}

Broth samples of $20 \mathrm{~mL}$ were taken from the $H$. akashiwo cultures in exponential and stationary phase conducted in flasks and PBRs. The cell pellets obtained by centrifuging the samples at $3000 \mathrm{~g}$ were extracted with $4 \mathrm{~mL}$ of methanol. The extracts were centrifuged at $10000 \mathrm{~g}$ for $5 \mathrm{~min}$, and the supernatants were stored in darkness at $20^{\circ} \mathrm{C}$ prior to use. The activity of the extracts was evaluated by the cell bioassay Neuro-2a (see M\&M Section 2.7). The extracts were subjected to gravity-flow purification. 500-mg C-18 columns were used, previously conditioned with HPLC grade methanol and deionized water. Subsequently, 1 to $1.5 \mathrm{~mL}$ of the methanolic extract was loaded. The sample was eluted with $100 \% \mathrm{MeOH}$, dried in a stream of nitrogen at $45^{\circ} \mathrm{C}$ and then resuspended in DMSO.

\subsection{Tandem mass spectrometric analysis of brevetoxins and brevenal}

The presence of brevetoxins (PbTx and BTX) and brevenal in the extracts was analyzed in a Waters XEVO TQ-XS tandem quadrupole atmospheric pressure (API) mass spectrometer (Waters, Eschborn, Germany) equipped with a high-performance ZSpray dual-orthogonal multi-mode (ESI/SPCI/ESCi) source, coupled to a Waters Acquity UPLC system consisting of a Waters Acquity UPLC I-Class Solvent Manager, a Waters Acquity UPLC I-Class flow-through-needle (FTN) Sample Manager, and a Acquity UPLC I-Class column manager (CM-A). Potential lipophilic toxins in the extracts were separated by injection $0.5 \mu \mathrm{L}$ of sample by reversed-phase chromatography on a C18 phase. The analytical column $(50 \times 2.1 \mathrm{~mm})$ was packed with $130 \AA$ and $1.7 \mu \mathrm{m}$ of $\mathrm{C} 18$, and maintained at $40{ }^{\circ} \mathrm{C}$. The flow rate was $0.6 \mathrm{~mL} \cdot \mathrm{min}^{-1}$ and gradient elution was performed with two eluants. Eluant $\mathrm{A}$ was water containing $0.01 \%$ formic acid and $0.05 \%$
Table 2

Mass transitions $(\mathrm{m} / z \mathrm{Q} 1>\mathrm{Q} 3)$ used to detect brevetoxins and brevenal

\begin{tabular}{lcc}
\hline Toxin & Q1 mass $(m / z)$ & Q3 mass $(m / z)$ \\
\hline PbTx-1 & 867.5 & 849.5 \\
PbTx-2 & 895.5 & 877.5 \\
PbTx-3 & 897.5 & 879.5 \\
PbTx-6 & 911.5 & 893.5 \\
PbTx-7 & 869.5 & 851.5 \\
PbTx-9 & 899.5 & 863.4 \\
PbTx-10 & 871.5 & 853.5 \\
BTX-B1 & 985.5 & 967.5 \\
BTX-B2 & 1034.6 & 1016.6 \\
Brevenal & 657.4 & 639.4 \\
\hline
\end{tabular}

ammonium hydroxide, and eluent B was acetonitrile containing $0.01 \%$ formic acid. Initial conditions were 0.5 min column equilibration with $30 \%$ eluant B, followed by a linear gradient to $90 \%$ B in 3 min and isocratic elution until $4 \mathrm{~min}$ with $90 \% \mathrm{~B}$. The system was then returned in $0.1 \mathrm{~min}$ to initial conditions (total run time: $5 \mathrm{~min}$ ).

Full scan experiments were performed in the mass range of $m / z 600$ to $m / z 1000$ in the positive ion mode. Mass spectrometric parameters were as follows: capillary voltage $3.5 \mathrm{kV}$, cone voltage $40 \mathrm{~V}$, desolvation temperature $600^{\circ} \mathrm{C}$, desolvation gas flow $1000 \mathrm{~L} \cdot \mathrm{h}^{-1}$, cone gas flow $150 \mathrm{~L}^{-1}$, nebuliser pressure $7.0 \mathrm{bar}$, collision gas flow $0.15 \mathrm{~mL} \cdot \mathrm{min}^{-1}$, collision $20 \mathrm{eV}$, and a source temperature of $150^{\circ} \mathrm{C}$.

Collision-induced dissociation (CID) produced ion spectra were recorded for the pseudomolecular ion of brevenal $\mathrm{m} / \mathrm{z} 657$. CID spectra were recorded with the same instrument in the ScanWave Daughter scan (ScanWave DS) modus in a mass range of $m / z 50$ to 660, and in a positive ionization and unit resolution mode. All mass spectrometric parameters were as described above for the full scan experiment. The mass transitions of brevetoxins and brevenal were set as detailed in Table 2 .

\subsection{Neuro-2a cell-based assay (CBA)}

The neuro-2a cytotoxicity bioassay, as reported by Astuya et al. [16] was used to detect brevetoxin-like neurotoxins acting on voltage-gated sodium channels (VGSCs). Briefly, neuro-2a mouse neuroblastoma cells (ATCC, CCL-131) were treated with the $\mathrm{Na}^{+} / \mathrm{K}^{+}$-ATPase inhibitor ouabain $(0.3 \mathrm{mM})$, the sodium channel-activator veratridine $(0.03 \mathrm{mM})$ and $H$. akashiwo extract $\left(12.5-100 \mu \mathrm{g} \cdot \mathrm{mL}^{-1}\right)$. The reduction of cytotoxicity in the presence of ouabain/veratridine $(0.3 / 0.003 \mathrm{mM})$, or "cell rescue", is indicative of sodium channel blocking or paralyzing toxins. The enhancement of cytotoxicity in the presence of ouabain/veratridine $(0.1 / 0.01 \mathrm{mM})$ is indicative of potent sodium channel activators like brevetoxin-like toxins [25-27]. The cytotoxicity of the $H$. akashiwo extract unrelated to the VGSC effect was ruled out by exposing neuro-2a cells to the extract in the absence of ouabain/veratridine. Cell viability was measured after $24 \mathrm{~h}$-incubation using MTT (3-(4,5-dimethylthiazol2-yl)-2,5-diphenyltetrazolium bromide, Life Technologies, Carlsbad, CA, USA) [25]. Absorbance was read at $570 \mathrm{~nm}$ using an automated multi-well scanning spectrophotometer (Synergy H1, Biotek, Vermont, USA). Cell viability was expressed as a percentage to control cells without the extracts ( $100 \%$ viability). Thus, the relative viability effect was represented as cell rescue. The cell rescue results of the neuro-2a $\mathrm{CBA}$ (presence of $\mathrm{O} / \mathrm{V}$ ) were expressed relative to the viability of cultures where saxitoxin standard $(50 \mathrm{nM})$ was used as a control. These control tests showed viabilities $>90 \%$. The standard was obtained from the Institute for Marine Biosciences, National Research Council of Canada. Concentrations of extracts of $100 \mathrm{mg} \cdot \mathrm{L}^{-1}$ were excluded since toxicity would mask cell rescue. Maximum cell rescue was obtained in the range of $25-50 \mathrm{mg} \cdot \mathrm{L}^{-1}$. 
2.8. Determination of the anaesthetic efficiency of the H. akashiwo methanolic extracts

Rearing, handling and experimental procedures were approved by the Bioethics Committee of the University of Concepción. A breeding stock of zebrafish (Danio rerio) wild type strain was maintained in accordance with international guidelines for zebrafish housing and husbandry; at $27 \pm 1{ }^{\circ} \mathrm{C}$, with a $12: 12 \mathrm{~h}$ light:dark photoperiod and constant water filtration. The fish were fed with Artemia sp. nauplii and TetraMin tropical flakes (Tetra, Hannover, Germany). To obtain the larvae needed for the experiments, spawning was induced daily and the healthy embryos were incubated at $27^{\circ} \mathrm{C}$ in embryo medium until hatching occurred. Concentrations of $H$. akashiwo methanolic extract ranging from 50 to $200 \mathrm{mg} \cdot \mathrm{L}^{-1}$ were previously assayed to define an adequate dose for anesthesia induction times below $5 \mathrm{~min}$. Zebrafish larvae at 7-8 days post fertilization (dpf) were exposed to $H$. akashiwo extract in wells (volume of $200 \mu \mathrm{L}$ ). The induction and recovery times of light anesthesia were then evaluated as described earlier [28]. Briefly, light anaesthetic stage is considered when there is partial loss of zebrafish larvae equilibrium and no reaction to external stimuli is observed. The results indicated that a concentration of $H$. akashiwo methanolic extract of around $200 \mathrm{mg} \cdot \mathrm{L}^{-1}$ induced anesthesia. This concentration was selected to evaluate anaesthetic activity of extracts prepared from PBR cultures. Induction and recovery times were measured for all larvae ( $N=5$ per experiment). Post-exposure survival was recorded $24 \mathrm{~h}$ after the experiments.

\subsection{Determination of in vitro cytotoxicity}

Antiproliferative assays were conducted as described elsewhere $[29,30]$ with crude methanolic extracts obtained from $H$. akashiwo biomass as were performed. Four human tumor cell lines obtained from the American Type Culture Collection (ATCC) were used, namely A549 (ATCC CCL-185) (lung carcinoma, NSCLC), HT-29 (ATCC HTB-38) (colon adenocarcinoma), MDA-MB-231 (ATCC HTB-26) (breast adenocarcinoma) and PSN-1 (ATCC CRL-3211) (pancreas adenocarcinoma). Cells were maintained in Dulbecco's modified Eagle's medium (DMEM), supplemented with $10 \%$ fetal bovine serum (FBS), $2 \mathrm{mM}_{\mathrm{L}-}$ glutamine, $100 \mathrm{U} / \mathrm{mL}$ penicillin and $100 \mathrm{U} / \mathrm{mL}$ streptomycin at $37^{\circ} \mathrm{C}$, $5 \% \mathrm{CO}_{2}$ and $98 \%$ humidity. Cells were harvested for the experiments from subconfluent cultures by trypsinization and resuspended in fresh medium before counting and plating. Human tumor cells were seeded in 96-well microtiter plates, at 5000 cells per well in aliquots of $150 \mu \mathrm{L}$ and allowed to attach to the plate surface for $18 \mathrm{~h}$ (overnight) in drugfree medium. One control (untreated) plate for each cell line was then fixed and used for the time zero reference value. Cells on plates were then exposed to different methanolic extract concentrations in DMSO/ DMEM $\left(10,30\right.$ and $\left.100 \mu \mathrm{gL}^{-1}\right)$. Cell survival was measured after treatment for $72 \mathrm{~h}$. Results are provided as survival percentages: $100 \%$ means there are as many cells as the control (extracts do not inhibit growth); $0 \%$ means that there is the same number of cells as at the start of the trial (no growth, extracts inhibit mitosis); $-100 \%$ means that all the cells were lysed (strong cytotoxic effect). Measurements were taken for triplicate samples. The standard deviations were in most cases $<$ $10 \%$.

\subsection{Statistics and data treatments}

All experimental data are presented as means \pm SD of three independent experiments performed with duplicate determinations. Data were treated and plotted using Prism 4.0 software (GraphPad Inc.).
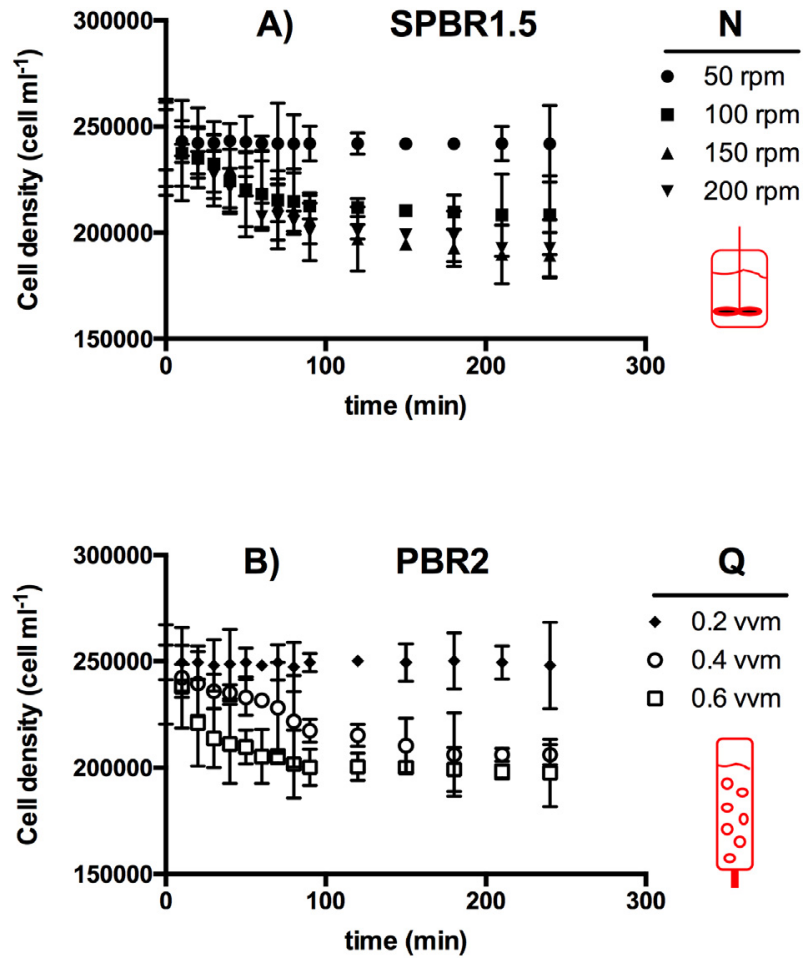

Fig. 1. Short-term effect of (A) the mechanical agitation rate (SPBR1.5), and (B) the aeration rate (PBR2) on $H$. akashiwo cell density. Each value is average of triplicate assays. Error bars represent standard deviation.

\section{Results and discussion}

\subsection{Damage energy dissipation rate threshold in agitated cultures of $H$.} akashiwo

Fig. 1 shows the growth kinetics of various short-term agitated $H$. akashiwo batch cultures. The disappearance of cells due to lysis was evident above an impeller speed $(N)$ of $50 \mathrm{rpm}$ with the mechanically agitated photobioreactor (SPBR1.5) (see Fig. 1A) and above an aeration rate of $0.2 \mathrm{v} \mathrm{v}^{-1} \mathrm{~min}^{-1}$ with the pneumatically agitated photobioreactor (PBR2) (see Fig. 1B). Based on the results shown in Fig. 1, the maximum specific death rates $\left(k_{\mathrm{d}}\right)$ were calculated with Eq. (6). Fig. 2 shows the $k_{d}$ values and corresponding EDR values (see Table 2). No EDR-associated damage was observed with either SPBR1.5 and PBR2 at similar EDR values, $1.8 \mathrm{~mW} \mathrm{~kg}^{-1}$ and $3.4 \mathrm{~mW} \mathrm{~kg}^{-1}$,

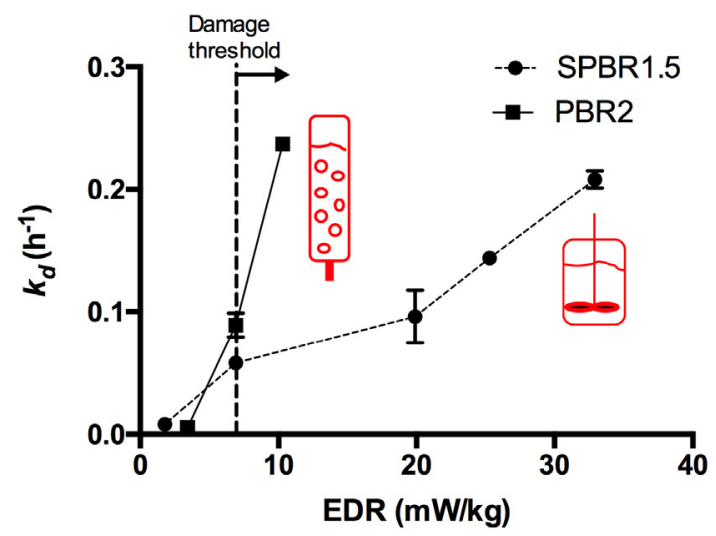

Fig. 2. Kinetic death rate constant $\left(k_{\mathrm{d}}\right)$ of $H$. akashiwo versus energy dissipation rate (EDR) for mechanical agitation (PBR1.5) and bubbling (PBR2) in benchscale photobioreactors. Each value is average of triplicate assays. Error bars represent standard deviation. 
respectively. As can be observed, $k_{d}$ increased with EDR in both PBRs, the increase in PBR2 being significantly higher than in PBR1.5. This difference is consistent with findings in similar shear sensitivity studies reported in the literature. In typical bioreactors, such as stirred tank and bubble column PBRs, cells are exposed to an inhomogeneous EDR field [31]. Local EDR values can be as much as two orders of magnitude higher in the impeller regions or on the liquid surface where bubbles break. Such high EDR values have been estimated to contribute around $70 \%$ of the total power in a typical bioreactor. The EDR fields and the proportion of both extensional flow and shear flow in stirred tanks and bubble columns are also entirely different at similar average EDRs. This means that the history and type of EDR experienced by cells in SPBR1.5 and PBR2 were probably different. The frequency of cell exposure to hydrodynamic extensional stress during the bubble bursting process in PBR12 is the probable cause of the higher $k_{\mathrm{d}}$ values than those in PBR1.5, in which cells were grown without bubbling. This extreme sensitivity of some microalgae to bubbling has been reported previously [5,32].

\subsection{Scale-up of Heterosigma akashiwo cell yield}

Fig. 2 shows EDR-associated damage thresholds for the two PBRs: between 1.8 and $6.9 \mathrm{~mW} \mathrm{~kg}^{-1}$ for PBR1.5, and between 3.4 and $6.9 \mathrm{~mW} \mathrm{~kg}^{-1}$ for PBR2. It is more difficult to maintain an average EDR in those ranges during PBR scale-up of PBRs with stirred reactors than with bubble columns because the mixing times in stirred reactors increase more rapidly with the scale size [31]. The percentage of the mixing power that is non-homogeneously dissipated near the stirrer increases with increases in scale size [33]. The EDR in the bubble columns can be kept at low levels by reducing the ratio of gas flow to culture volume. Consequently, the operational $E D R$ values for the PBR12L and PBR200L cultures were established at 5.3 and $5.1 \mathrm{~mW} \mathrm{~kg}^{-1}$ by setting gas flow rates at 0.042 and $0.025 \mathrm{v} \mathrm{v}^{-1} \mathrm{~min}^{-1}$, respectively, both equivalent to a surface gas velocity of 5.4 $10^{-4} \mathrm{~m} \mathrm{~s}^{-1}$. These flow rates allowed the cells to be homogeneously distributed in the PBR. However, degassing and the average time required for a single cell to travel from dark to illuminated areas were clearly lower in the larger PBR.

The scale-up conditions described above were compared in terms of biomass yield for the photoautotrophic growth of $\mathrm{H}$. akashiwo. Two types of culture devices of three sizes were used: (i) static culture (without agitation) in a 1-L Erlenmeyer flask in which $\mathrm{CO}_{2}$ was provided by diffusion through a culture-air interface; and (ii) pneumatically-agitated cultures in the PBR12 and PBR200, in which mixing and $\mathrm{CO}_{2}$ was provided via air bubbling in the culture medium. $\mathrm{CO}_{2}$ was not controlled in the PBRs in order to observe mainly the effect of EDR during the scale-up process. The PBR200 also allowed us to determine if growth limitation caused by $\mathrm{CO}_{2}$ and/or light had an impact on the bioactive production. Table 3 shows the corresponding surface-to-volume ratios in decreasing order in the culture devices. Fig. 3 shows the growth kinetics of $H$. akashiwo. Maximum specific growth rates in the exponential phase were different among the three culture systems (see Table 3). Kinetics converged at a stationary final concentration around 300,000 cell $\mathrm{mL}^{-1}$ in the flask and PBR12 cultures, while a lower cell

Table 3

Kinetic parameters determined from results of Heterosigma akashiwo batch cultures grown in PBRs of different scales: maximum specific growth rate, $\mu_{\text {max }}$; maximum doubling time, $T_{\mathrm{d}} ; \mathrm{S} / \mathrm{V}$ is the surface-to-volume ratio. Each value is average of triplicate assays.

\begin{tabular}{lllll}
\hline PBR & S/V ratio & Mixing & $\mu_{\max }\left(\right.$ day $\left.^{-1}\right)$ & $T_{\mathrm{d}}$ (day) \\
\hline Flask & 4.75 & Static & 0.264 & 2.446 \\
PBR12 & 1.28 & Bubbling & 0.36 & 1.882 \\
PBR200 & 0.79 & Bubbling & 0.12 & 5.385 \\
\hline
\end{tabular}

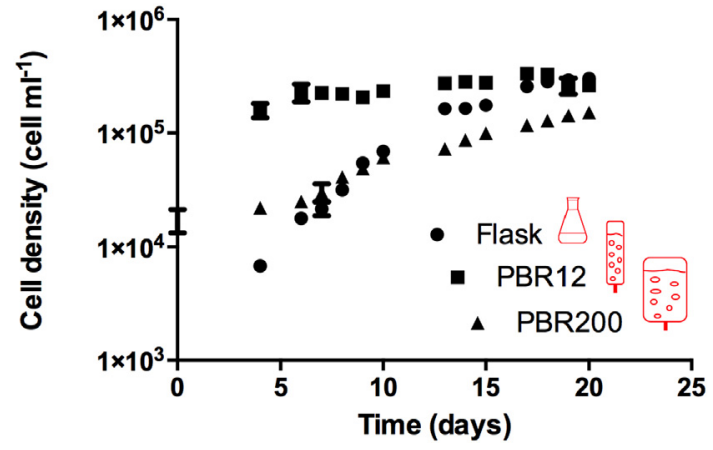

Fig. 3. Effect of scale-up on the cell yield of Heterosigma akashiwo grown in different photobioreactors. Each value is average of triplicate assays. Error bars represent standard deviation.

concentration of approximately 150,000 cell $\mathrm{mL}^{-1}$ was reached in PBR200.

The slowest doubling time $\left(\mu_{\max }\right)$ was with the PBR200, while the most rapid was with the PBR12 (Table 3). Doubling required nearly two days with the PBR12. These results were expected in photosynthetic cultures due to the advantages of bubbling columns with a high heightto-diameter ratio compared to those with lower ratios [34]. The light availability was maximum for the PBR12 because of a shorter light path. A shorter light path with the same incident irradiance supposes less auto-shading. PBR200 may have been limited by available light since exponential growth was slower. However, the low air flow rate fixed in the PBR200 suggests that $\mathrm{CO}_{2}$ limitation was also an issue during most the culture period. Evidence for this is the linear growth phase of the PBR200 culture from the thirteenth day to the end of the culture in Fig. 3. Only one or both of the two nutrients that were continuously supplied to the culture $\left(\mathrm{CO}_{2}\right.$ at $0.03 \% \mathrm{vol} / \mathrm{vol}$ in the aeration gas and light) could have caused it and therefore limited growth. That is, the linear growth phase was established when $\mathrm{CO}_{2}$ and/or light energy consumptions balanced the $\mathrm{CO}_{2}$ and photons transfer. To establish if the limiting process was the $\mathrm{CO}_{2}$ transfer, an approach based on characteristic time analysis was used as reported elsewhere [35]. Thus, two different characteristic times, such as mass transfer time $\left(t_{M T}\right)$ and $\mathrm{CO}_{2}$ consumption time $\left(t_{C}\right)$, were taken into account. The characteristic mass transfer time for $\mathrm{CO}_{2}$ was evaluated as:

$t_{M T}=\frac{1}{K_{L} a}$

where $K_{L} a$ is the volumetric mass transfer coefficient for $\mathrm{CO}_{2}$. For a $u_{g}$ of $5.410^{-4} \mathrm{~m} \mathrm{~s}^{-1}$ in the PBR2000, a $K_{L} a$ value of $3.3210^{-4} \mathrm{~s}^{-1}$ was obtained from the following equation reported for seawater in pilot bubble column PBRs [36]:

$K_{L} a=\frac{2.222}{u_{g}^{-1.171}-1}$

Thus, from Eq. (7) a $t_{M T}$ value of $3020 \mathrm{~s}$ was calculated. During growth, $t_{C}$ was expressed as

$t_{C}=\frac{C_{C O 2}^{*}}{\frac{1}{Y_{C O 2}} \mu C_{b}}$

where $\mathrm{C}_{\mathrm{CO} 2}{ }^{*}$ is the equilibrium concentration of $\mathrm{CO}_{2}$ with a value estimated of $0.45 \mathrm{~g} \mathrm{~m}^{-3}$ at $20^{\circ} \mathrm{C}, C_{b}$ is the biomass concentration expressed in terms of dry weight $\left(\mathrm{g} \mathrm{m}^{-3}\right)$ and $\mathrm{Y}_{\mathrm{CO} 2}$ is the $\mathrm{CO}_{2}$ yield coefficient (a value of 0.55 was assumed) [35]. The conversion of the cell concentration data $(N)$ for PBR200 in Fig. 3 to $C_{b}$ was performed from the existing correlation between $C_{b}$ and cell biovolume $\left(V_{\text {cell }}\right)$ [7]:

$C_{b}=\xi \cdot N \cdot V_{\text {cell }}$

where $V_{\text {cell }}$ was calculated from the average equivalent mean cell diameter $\left(1410^{-6} \mathrm{~m}\right)$ measured by flow cytometry. A conservative $\xi$ value 
has been recently suggested to be around $0.180 \mathrm{~g}$ d.w. $\mathrm{mL}^{-1}$ in basis to a fresh microalgal cells density of $1.2 \mathrm{~g} \mathrm{~mL}^{-1}$ and a dry mass fraction of fresh cells of $25 \%$ [7]. In linear phase, the product $\mu \cdot C_{b}$ in Eq. (10) is constant with a value of $4.7 \mathrm{~g} \mathrm{~m}^{-3} \mathrm{day}^{-1}$. Thus, the characteristic consumption time for $\mathrm{CO}_{2}, t_{C}$, estimated from Eq. (9) was of $190 \mathrm{~s}$. As $t_{C}$ was clearly lower than $t_{M T}, \mathrm{CO}_{2}$-consumption was controlled by $\mathrm{CO}_{2^{-}}$ transfer from the gas phase. Regarding the light, the daily mean volumetric photon flux absorbed by the culture $(\Gamma)$ was evaluated to determine if the linear phase in the PBR200 culture was co-limited by light. Because the photons received by the walls of the PBR200 were virtually absorbed by the culture in the linear phase (i.e. the light path in the PBR200 and cell concentrations achieved in the linear phase were such that no photonic flow escaped from the PBR200 wall), $\Gamma$ have to be constant, independent of $C_{b}$ and close to the maximum photon flux potentially absorbable by the culture (i.e. $\Gamma_{\max }$ ). Assuming a two-dimensional totally diffuse incident light, $\Gamma_{\max }$ can be estimated from the following expression [8]:

$\Gamma_{\max }=\frac{2 I_{o}}{R \pi}$

where $I_{o}\left(100 \mu \mathrm{E} \mathrm{m}^{-2} \mathrm{~s}^{-1}\right)$ is the irradiance measured in the center of the PBR200 filled with seawater and $R\left(22.510^{-2} \mathrm{~m}\right)$ is the PBR200 radius. The value of $\Gamma_{\max }$ calculated was $283 \mu \mathrm{E} \mathrm{m}^{-3} \mathrm{~s}^{-1}$, a more than three-fold lesser relative to PBR12. The value of $C_{b}$ at which $\Gamma_{\max }$ is virtually reached in PBR200 is close to $0.031 \mathrm{~kg} \mathrm{~m}^{-3}$ on day 13 , which is the biomass concentration where linear growth phase starts. Therefore, the irradiance supplied to PBR200 seemed to be too low and potentially co-responsible for the appearance of the linear growth phase. This is also supported by previous results obtained with other microalgae. For example, the linear growth phase of an airlift PBR culture of Phaedactylum tricornutum commenced with a $C_{b}$ near $1.2 \mathrm{~kg} \mathrm{~m}^{-3}$ when a $\Gamma_{\max }$ of $16,500 \mu \mathrm{E} \mathrm{m}^{-3} \mathrm{~s}^{-1}$ was absorbed by the culture [35]. In the case of Amphidinium carterae, a $\Gamma_{\max }$ value of $2510 \mu \mathrm{E} \mathrm{m} \mathrm{m}^{-3} \mathrm{~s}^{-1}$ caused a linear growth phase at close to $C_{b} 0.2 \mathrm{~kg} \mathrm{~m}^{-3}$ [7]. For a $\Gamma_{\max }$ of $283 \mu \mathrm{E}$ $\mathrm{m}^{-3} \mathrm{~s}^{-1}$ as that measured in the PBR200, both aforementioned microalgae would have hypothetically commenced their linear growth phase at around $0.02 \mathrm{~kg} \mathrm{~m}^{-3}$, in line with that above estimated for $H$. akashiwo in the PBR200.

There have been few reports on pilot-plant scale culturing of the ichthyotoxic microalgae $H$. akashiwo, although there are a number of works cataloguing this species as a producer of interesting bioactives $[16,19,37]$. Fuentes-Grünewald et al. [38] provided an example of intensive culture of an $H$. akashiwo strain. However, research has generally been performed with uncontrolled cultures at laboratory scales with volumes not exceeding a liter. At this scale, $H$. akashiwo grows optimally $[15,39]$. As in the present work, volumetric cell productivity was higher, and the maximum specific growth rate was greater in the PBR12.

The scale-up to PBR200 based on maintaining the optimal $Q$ value constant for PBR12 (i.e. $0.042 \mathrm{v} \mathrm{v}^{-1} \mathrm{~min}^{-1}$ ) implied setting EDR at approximately $8.85 \mathrm{~mW} \mathrm{~kg}^{-1}$ in PBR200. Nevertheless, this EDR produced cell lysis in PBR2 (see Fig. 2). There were also noticeable differences in the aspect ratio (i.e. length-to-diameter ratio) among the bioreactors: 5.6 in PBR12 and 2.8 in PBR200. The interaction of PBR hydrodynamics and geometry ( $\mathrm{S} / \mathrm{V}$ ratio and aspect ratio) provided a more efficient illumination and $\mathrm{CO}_{2}$-transfer regime than that in PBR200. Optimal growth of highly shear-susceptible microalgae in bubble column PBRs is complex because varied design and operational parameters have to be carefully selected to minimize turbulence-associated damage to cells [40]. Air flow rate alone should not be used to improve cell growth and mitigate damage in sparged photobioreactors because other parameters such as $\mathrm{CO}_{2}$ molar fraction in the injected air, column volume, sparger geometry details, and mass transfer, should also be taken into consideration. A model reported for shear-sensitive dinoflagellate microalgae provides guidance on how several of these types of parameters can be manipulated to reach adequate mass transfer



B

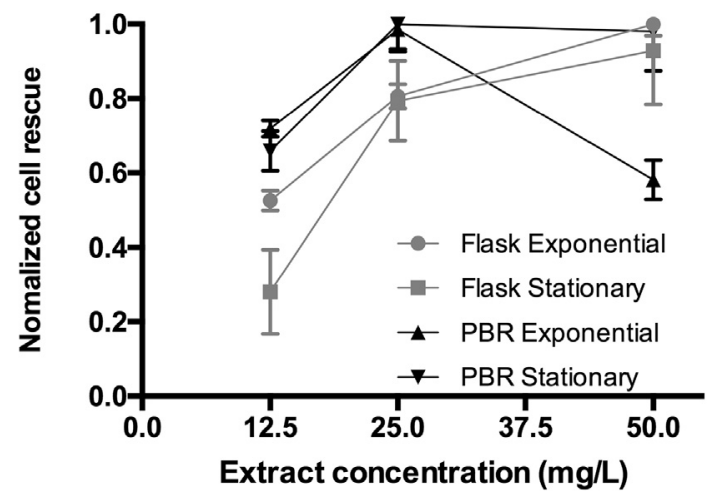

Fig. 4. A) \% Viability of neuro-2a CBA with Heterosigma akashiwo methanolic extracts prepared from biomass harvested in exponential and stationary growth phases from flask and PBR12 cultures; B) Normalized cell rescue (increase in viability) of neuro-2a cells exposed to ouabaine/veratridine and $H$. akashiwo extracts. A value of 1 represents recovery of total viability using saxitoxin standard $(50 \mathrm{nM})$. Each value is average of triplicate assays. Error bars represent standard deviation.

and to avoid cell damage [40,41]. In principle, pneumatically-agitated culture systems like bubble columns are candidate PBRs for the production of bioactives from $H$. akashiwo. Any further scale up of production should rely on increasing the number of PBRs. Future research should be aimed at optimizing the PBR unit to be used.

\subsection{Functional biological characterization of $H$. akashiwo extracts}

Neurotoxins acting on VGSCs were assessed according to the protocol described by [16]. First, we evaluated the cytotoxic effect of the extract of $H$. akashiwo on the neuro-2a model and then determined the "rescue activity" caused by a blockage of the $\mathrm{Na}^{+}$channel. The ability to block the $\mathrm{Na}^{+}$channel is similar to that of TTX or brevenal, which have been shown to produce anesthesia $[16,42]$. The first assessment was carried out to determine cytotoxicity unrelated to any effect on the $\mathrm{Na}^{+}$channel. Fig. 4A shows the percentages of viable neuro-2a cells exposed to $H$. akashiwo methanolic extracts ranging from 12.5 to $100 \mathrm{mg} \mathrm{L}^{-1}$. Extracts were prepared from biomass harvested in the exponential and stationary phases from the cultures in PBR12 and the Erlenmeyer flask. There were evident cytotoxic effects in extracts from cultures in PBR12 and the flasks at concentrations of 50 and $100 \mathrm{mg} \mathrm{L}^{-1}$, respectively. The mortality rate was $100 \%$ in the PBR12 culture extracts of the exponential and stationary growth phases. The viability of flask-grown neuro-2a cells from the exponential and stationary phases decreased respectively by 30 and $50 \%$. Although the cytotoxic effects on the cultures grown in flasks and PBR12 were similar, the effects on extracts from the PBR12 culture were much greater, with little difference among growth phases. For the flask 
cultures, the extract obtained during the exponential growth phase had a greater impact on viability than the stationary one.

Interestingly, the $100 \mathrm{mg} \cdot \mathrm{L}^{-1}$ extract from PBR12 did not show cytotoxic effects against four human tumor cell lines typically used in standard antiproliferative assays. This indicates that the cytotoxic effect on neuro-2a cells cannot be extrapolated to other cell lines. This is confirmed by Fig. 4B, which shows the experimental data of cellular rescue produced by the Heterosigma akashiwo extracts when the $\mathrm{O} / \mathrm{V}$ opens the $\mathrm{Na}^{+}$channel. The results indicate a biological effect of the extracts similar to those of other paralyzing marine biotoxins [16]. However, Fig. 4B shows a deviation in this pattern for the $50 \mathrm{mg} \cdot \mathrm{L}^{-1}$. extract from the PBR12-exponential biomass because there was less rescue activity.

A brevetoxin-like bioactive produces cell death rather than cell rescue in the presence of $\mathrm{O} / \mathrm{V}$ (Fig. 4B). In that case, negative values of normalized cell rescue are obtained. Overall, the results shown in Fig. 4B indicate that the compounds in the extracts from the PBR12 and flask cultures with bioactivity related to the $\mathrm{Na}^{+}$channel are similar. Saxitoxin-type bioactives seem the most likely candidates. Assuming that the viability values in Fig. 4A are a proxy for concentration of saxitoxin-type compounds in the extracts, the effect of growth phase and PBR type on the viability of neuro-2a cells confirms that the biosynthesis of this kind of bioactives is a complex process, as reported for other microalgae [44].

\subsection{Chemical analysis of the H. akashiwo extract}

Although functional assays have clearly shown that extracts of $H$. akashiwo have brevenal-like activity, nothing is known about the identity of the chemical compounds eliciting these effects. However, several studies have reported the presence of brevetoxins in raphidophytes $[19,44,45]$. Astuya et al. [16] reported the presence of a paralyzing toxin (HaTx) in $H$. akashiwo extracts that block or inhibit voltage-dependent sodium-channels (VDSCs), a behavior similar to those of saxitoxin and tetrodotoxin, but also to that of brevenal, which acts as an antagonist of brevetoxins [4].

In the light of these reports, the extracts of the raphidophyte $H$. akashiwo were analyzed for the presence of brevetoxins and brevenal. None of the brevetoxins listed in Table 1 were detected in any of the analyzed CCMP302 H. akashiwo extracts. The detection limit (S/ $\mathrm{N}>3$ ) was calculated as $0.1 \mathrm{fg}^{\text {cell }}{ }^{-1}$ for PbTx- 2 and was in the same order of magnitude for the other brevetoxins, of which standard solutions were available (PbTx-3, -6 and -9$)$. The absence of brevetoxins has also been reported for the raphidophyte Fibrocapsa japonica [20], and raises questions about previous reports of the production of brevetoxins by raphidophytes. In contrast to the absence of any known brevetoxins, all extracts showed a brevenal peak at mass transition $(\mathrm{m} / \mathrm{z}$ $657.4>639.4)$ at the retention time of $3.5 \mathrm{~min}$. As there was no brevenal standard solution available for compound identification, a collision induced dissociation (CID) spectrum was recorded to obtain more detailed information about this compound. The pseudo-molecular ion was the base peak of the CID spectrum of the $m / z 657 \mathrm{H}$. akashiwo compound, with very few fragments. The second most abundant fragment was water loss from the pseudo-molecular ion and smaller fragments in low abundance at $m / z 109,121,149$ and 215. In contrast, the spectrum of brevenal was characterized by six consecutive water loss events from the pseudo-molecular ion, which is very characteristic of polyketides like brevenal, and a few of smaller fragments, of which the most abundant were $m / z 159$ and 255. The discrepancies among the fragments of brevenal and the isobaric compound in H. akashiwo are clear evidence that $H$. akashiwo does not produce brevenal and that the isobaric compound found in $H$. akashiwo is not chemically related to polyketides of the brevetoxin group. The absence of brevetoxins and brevenal in the $H$. akashiwo extracts shows that $H$. akashiwo produces chemical compound(s) that are structurally unrelated to brevetoxins, but that elicit similar biological functions. The lack of knowledge about

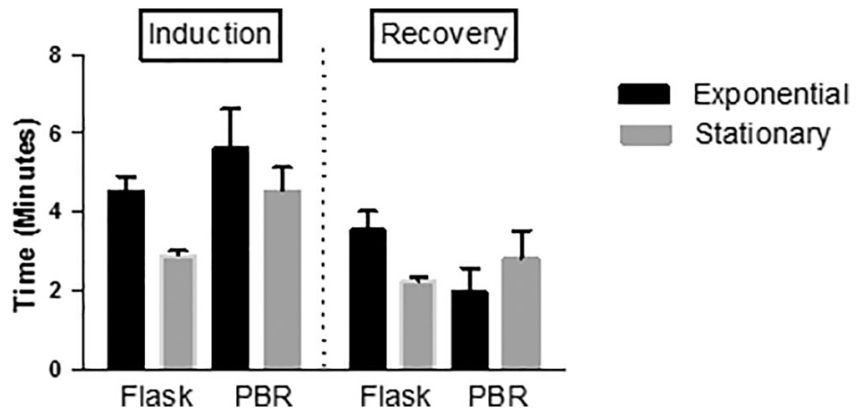

Fig. 5. Light anesthesia induction and recovery times in Zebra fish larvae produced by Heterosigma akashiwo methanolic extracts $\left(200 \mathrm{mg} \cdot \mathrm{L}^{-1}\right)$ prepared from biomass harvested from flask and PBR12 cultures in the exponential and stationary growth phases. Each value is average of triplicate assays. Error bars represent standard deviation.

secondary bioactive metabolites of raphidophytes and other ichthyotoxic species clearly demonstrates the need for further research in this field.

\subsection{Anaesthetic efficiency}

The in vivo anaesthesic effect of $H$. akashiwo extracts was evaluated with the zebrafish larval model. Zebrafish have been widely used in the evaluation of anaesthetics, both from the perspective of animal care $[46,47]$ and the search for optimal anaesthetics for vertebrates [48]. Recently, zebrafish larvae have been proposed for testing analgesic efficacy and nociception [49,50], given that larvae have a similar response to a noxious challenge to that of adult zebrafish and other vertebrates. Induction and recovery times in our assays were below $6 \mathrm{~min}$ when extracts of $200 \mathrm{mg} \cdot \mathrm{L}^{-1}$ were used (Fig. 5). We did not observe mortality for $24 \mathrm{~h}$ after exposure. There were differences in anaesthetic induction times based on culture methods and phases, with the shortest times in extracts from flask cultures harvested in the stationary phase (Fig. 5). Recovery times were lower in extracts from the PBR12 culture (ANOVA $\mathrm{p}<0.001$ ) in stationary phase. Nevertheless, differences remained low (Fig. 5). Although there were significant differences in anaesthetic efficiency between culture methods and phase of cultivation, the ranges of induction and recovery times showed that culture in the PBR12 did not change the ability of the extracts to induce anesthesia. The induction and recovery times were close to those desired for an anaesthetic agent (induction $3 \mathrm{~min}$, recovery 5 min [28]). As well, the same quantified order of magnitude was observed for zebrafish [51] and species of commercial importance [52] as with anaesthetics currently in use.

\section{Conclusions}

Cultures of $H$. akashiwo were grown in PBRs of up to $200 \mathrm{~L}$ at an EDR of $5.1 \mathrm{~mW} \mathrm{~kg}^{-1}$ with a gas flow of $0.025 \mathrm{v} \mathrm{v}^{-1} \mathrm{~min}^{-1}$. Under these conditions, we observed a homogeneous distribution of microalgal cells in the PBRs and the absence of hydrodynamic damage. However, growth was optimal in the PBR12 culture due to the narrower light path and better gassing/degassing. Chemical analysis of the biomass revealed that $H$. akashiwo produces chemical compounds unrelated structurally to brevetoxins or brevenal. The methanolic extract of harvested biomass did not show cytotoxic (up to $50 \mathrm{mg} \cdot \mathrm{L}^{-1}$ ) or saxitoxinlike effects. PBR cultures produced biomasses with similar characteristics in the different growth phases, although the stationary phase showed higher toxicity in the neuro-2a cellular model. However, cytotoxicity evaluated on four human tumor cell lines showed that the crude methanolic extract did not present cytotoxicity. The anaesthetic effect of the methanolic extract, assessed according to the Zebra fish model, was similar to those of commercial products, and without 
observed mortality at 24-h post-exposure.

"No conflicts, informed consent, or human rights are applicable to this study. Animal rights: Rearing, handling and experimental work with zebrafish larvae were carried out under protocols approved by Bioethics Committee of University of Concepción and in accordance with internationally established procedures."

\section{Declaration of competing interest}

The authors declare that:

o All authors have participated in (a) conception and design, or analysis and interpretation of the data; (b) drafting the article or revising it critically for important intellectual content; and (c) approval of the final version.

o This manuscript has not been submitted to, nor is under review at, another journal or other publishing venue.

o The authors have no affiliation with any organization with a direct or indirect financial interest in the subject matter discussed in the manuscript.

o The authors have no other conflict of interests regarding the submitted manuscript.

\section{Acknowledgements}

This research was mainly funded by the projects Fondecyt 1170515 (Conicyt, Chile) and Fondef IDEA 2017 ID17I10100 (Conicyt, Chile). This research has been also partially funded by the State Research Agency (grants RTC-2017-6405-1 and CTQ2014-55888-C3-02 (MARBIOM)) of the Ministry of Science, Innovation and Universities and the European Regional Development Fund Program and by the Helmholtz-Gemeinschaft Deutscher Forschungszentren through the research programme PACES II of the Alfred Wegener Institut-Helmholtz Zentrum für Polar- und Meeresforschung. We would also like to thank Dr. Fernando de la Calle Verdú (PharmaMar S.A., Spain) by performing the antiproliferative assays.

\section{References}

[1] J. Assunção, A. Catarina Guedes, F. Xavier Malcata, Biotechnological and pharmacological applications of biotoxins and other bioactive molecules from dinoflagellates, Mar. Drugs 15 (2017), https://doi.org/10.3390/md15120393.

[2] J.J. Gallardo-Rodríguez, A. Sánchez-Mirón, F. García-Camacho, L. López-Rosales, Y. Chisti, E. Molina-Grima, Bioactives from microalgal dinoflagellates, Biotechnol. Adv. 30 (2012) 1673-1684, https://doi.org/10.1016/j.biotechadv.2012.07.005.

[3] R. Zhang, C. Li, J. Wang, Y. Yang, Y. Yan, Microbial production of small medicinal molecules and biologics: from nature to synthetic pathways, Biotechnol. Adv. 36 (2018) 2219-2231, https://doi.org/10.1016/j.biotechadv.2018.10.009.

[4] A.J. Bourdelais, S. Campbell, H. Jacocks, J. Naar, J.L.C. Wright, J. Carsi, D.G. Baden, Brevenal is a natural inhibitor of brevetoxin action in sodium channel receptor binding assays, Cell. Mol. Neurobiol. 24 (2004) 553-563, https://doi.org/ 10.1023/B:CEMN.0000023629.81595.09.

[5] F. García Camacho, J.J. Gallardo Rodríguez, A. Sánchez Mirón, M.C. Cerón García, E.H. Belarbi, E. Molina Grima, Determination of shear stress thresholds in toxic dinoflagellates cultured in shaken flasks, Process Biochem. 42 (2007) 1506-1515, https://doi.org/10.1016/j.procbio.2007.08.001.

[6] F.G. Camacho, J.J.G. Rodríguez, A.S. Mirón, E.H. Belarbi, Y. Chisti, E.M. Grima, Photobioreactor scale-up for a shear-sensitive dinoflagellate microalga, Process Biochem. 46 (2011) 936-944, https://doi.org/10.1016/j.procbio.2011.01.005.

[7] A. Molina-Miras, A. Morales-Amador, C.R. de Vera, L. López-Rosales, A. SánchezMirón, M.L. Souto, J.J. Fernández, M. Norte, F. García-Camacho, E. Molina-Grima, A pilot-scale bioprocess to produce amphidinols from the marine microalga Amphidinium carterae: isolation of a novel analogue, Algal Res. 31 (2018) 87-98, https://doi.org/10.1016/j.algal.2018.01.010.

[8] L. López-Rosales, F. García-Camacho, A. Sánchez-Mirón, E. Martín Beato, Y. Chisti, E. Molina Grima, Pilot-scale bubble column photobioreactor culture of a marine dinoflagellate microalga illuminated with light emission diodes, Bioresour. Technol. 216 (2016) 845-855, https://doi.org/10.1016/j.biortech.2016.06.027.

[9] J.J. Gallardo Rodríguez, A. Sánchez Mirón, F. García Camacho, M.C. Cerón García, E.H. Belarbi, E. Molina Grima, Culture of dinoflagellates in a fed-batch and continuous stirred-tank photobioreactors: growth, oxidative stress and toxin production, Process Biochem. 45 (2010) 660-666, https://doi.org/10.1016/j.procbio. 2009.12.018.
[10] J.J. Gallardo-Rodríguez, A. Astuya-Villalón, A. Llanos-Rivera, V. Avello-Fontalba, V. Ulloa-Jofré, A critical review on control methods for harmful algal blooms, Rev. Aquac. (2018) 1-24, https://doi.org/10.1111/raq.12251.

[11] Y. Fukuyo, I. Imai, M. Kodama, K. Tamai, Red tides and other harmful algal blooms in Japan, in: F.J.R. Taylor, V.L. Trainer (Eds.), Harmful Algal Bloom, PICES Reg. North Pacific, North Pacific Marine Science Organization (PICES), Sidney, 2002, pp. $7-20$.

[12] A.L. Clement, Phytoplankton monitoring program in the fish farming region of south Chile, in: T.J.S. Smayda (Ed.), Toxic Phytoplankt. Bloom. Sea, Elsevier Science Publishers B.V., Amsterdam, 1993, pp. 223-258.

[13] J.-S. Ki, M.-S. Han, Nuclear rDNA and chloroplast rbcL, rbcS and IGS sequence data, and their implications from the Japanese, Korean, and North American harmful algae, Heterosigma akashiwo (Raphidophyceae), Environ. Res. 103 (2007) 299-304, https://doi.org/10.1016/j.envres.2006.08.014.

[14] J.J. Dorantes-Aranda, A. Seger, J.I. Mardones, P.D. Nichols, G.M. Hallegraeff, Progress in understanding algal bloom-mediated fish kills: the role of superoxide radicals, phycotoxins and fatty acids, PLoS One 10 (2015), https://doi.org/10. 1371/journal.pone.0133549.

[15] A. Astuya, A. Rivera, K. Vega-Drake, C. Aburto, F. Cruzat, V. Ulloa, T. Caprile, J.J. Gallardo-Rodríguez, Study of the ichthyotoxic microalga Heterosigma akashiwo by transcriptional activation of sublethal marker Hsp70b in Transwell co-culture assays, PLoS One 13 (2018), https://doi.org/10.1371/journal.pone.0201438.

[16] A. Astuya, A.E. Ramírez, A. Aballay, J. Araya, J. Silva, V. Ulloa, J. Fuentealba, Neurotoxin-like compounds from the ichthyotoxic red tide alga Heterosigma akashiwo induce a TTX-like synaptic silencing in mammalian neurons, Harmful Algae 47 (2015) 1-8, https://doi.org/10.1016/j.hal.2015.04.006.

[17] M. Endo, Y. Onoue, A. Kuroki, Neurotoxin-induced cardiac disorder and its role in the death of fish exposed to Chattonella marina, Mar. Biol. 112 (1992) 371-376, https://doi.org/10.1007/BF00356281.

[18] J.H. Landsberg, The effects of harmful algal blooms on aquatic organisms, Rev. Fish Sci. 10 (2002) 113-390, https://doi.org/10.1080/20026491051695.

[19] S. Khan, O. Arakawa, Y. Onoue, Neurotoxins in a toxic red tide of Heterosigma akashiwo (Raphidophyceae) in Kagoshima Bay, Japan, Aquac. Res. 28 (1997) 9-14, https://doi.org/10.1046/j.1365-2109.1997.t01-1-00823.x.

[20] M.K. de Boer, C. Boerée, S.B. Sjollema, T. de Vries, A.D. Rijnsdorp, A.G.J. Buma, The toxic effect of the marine raphidophyte Fibrocapsa japonica on larvae of the common flatfish sole (Solea solea), Harmful Algae 17 (2012) 92-101, https://doi. org/10.1016/j.hal.2012.03.005.

[21] C.J. Keppler, A.J. Lewitus, A.H. Ringwood, J. Hoguet, T. Staton, Sublethal cellular effects of short-term raphidophyte and brevetoxin exposures on the eastern oyster Crassostrea virginica, Mar. Ecol. Prog. Ser. 312 (2006) 141-147, https://doi.org/ 10.3354/meps312141.

[22] R.R.L. Guillard, P.E. Hargraves, Stichochrysis immobilis is a diatom, not a chyrsophyte, Phycologia 32 (1993) 234-236.

[23] S. Ruszkowski, A rational method for measuring blending performance, and comparison of different impeller types, Proc. 8th Eur. Conf. Mix, 1994, pp. 283-291.

[24] Y. Chisti, Shear sensitivity, Encycl. Bioprocess Technol. Ferment. Biocatal. Biosep, Wiley, New York, 1999, pp. 2379-2406 http://www.scopus.com/inward/record. url? eid = 2-s2.0-0000037343\&partnerID $=$ tZOtx3y1.

[25] R.L. Manger, L.S. Leja, S.Y. Lee, J.M. Hungerford, M.M. Wekell, Tetrazolium-based cell bioassay for neurotoxins active on voltage-sensitive sodium channels: semiautomated assay for saxitoxins, brevetoxins, and ciguatoxins, Anal. Biochem. 214 (1993) 190-194, https://doi.org/10.1006/abio.1993.1476.

[26] E. Cañete, J. Diogène, Comparative study of the use of neuroblastoma cells (Neuro2a) and neuroblastoma $\times$ glioma hybrid cells (NG108-15) for the toxic effect quantification of marine toxins, Toxicon 52 (2008) 541-550, https://doi.org/10. 1016/j.toxicon.2008.06.028.

[27] A. Caillaud, P. de la Iglesia, H.T. Darius, S. Pauillac, K. Aligizaki, S. Fraga, M. Chinain, J. Diogène, Update on methodologies available for ciguatoxin determination: perspectives to confront the onset of ciguatera fish poisoning in Europe, Mar. Drugs 8 (2010) 1838-1907, https://doi.org/10.3390/md8061838.

[28] L.G. Ross, B. Ross (Eds.), Anaesthetic and Sedative Techniques for Aquatic Animals, Third, Wiley, New York, 2008, , https://doi.org/10.1002/9781444302264.

[29] C. Schleissner, L.M. Cañedo, P. Rodríguez, C. Crespo, P. Zúñiga, A. Peñalver, F. de la Calle, C. Cuevas, Bacterial production of a pederin analogue by a free-living marine alphaproteobacterium, J. Nat. Prod. 80 (2017) 2170-2173.

[30] R.H. Shoemaker, The NCI60 human tumour cell line anticancer drug screen, Nat. Rev. Cancer 6 (2006) 813.

[31] K. van't Riet, R.G.J.M. van der Lans, Mixing in bioreactor vessels, in: M. Moo-Young (Ed.), Compr. Biotechnol, second ed., Elsevier B.V., 2011, pp. 63-80, , https://doi. org/10.1016/B978-0-08-088504-9.00083-0 Second Edi.

[32] L. López-Rosales, F. García-Camacho, A. Sánchez-Mirón, A. Contreras-Gómez, E. Molina-Grima, An optimisation approach for culturing shear-sensitive dinoflagellate microalgae in bench-scale bubble column photobioreactors, Bioresour. Technol. 197 (2015) 375-382, https://doi.org/10.1016/j.biortech.2015.08.087.

[33] J. Varley, J. Birch, Reactor design for large scale suspension animal cell culture, Cytotechnology 29 (1999) 177-205, https://doi.org/10.1023/A:1008008021481.

[34] A. Sánchez Mirón, A.C. Gómez, F.G. Camacho, E.M. Grima, Y. Chisti, Comparative evaluation of compact photobioreactors for large-scale monoculture of microalgae, Prog. Ind. Microbiol. 35 (1999) 249-270, https://doi.org/10.1016/S00796352(99)80119-2.

[35] A. Contreras, F. García, E. Molina, J.C. Merchuk, Interaction between CO2-mass transfer, light availability, and hydrodynamic stress in the growth of Phaeodactylum tricornutum in a concentric tube airlift photobioreactor, Biotechnol. Bioeng. 60 (1998) 317-325.

[36] A.S. Mirón, F.G. Camacho, A.C. Gómez, E.M. Grima, Y. Chisti, Bubble-column and 
airlift photobioreactors for algal culture, AICHE J. 46 (2000) 1872-1886 https:// www.scopus.com/inward/record.uri?eid $=2$-s2.0-0034281532\&partnerID $=40 \&$ md5 = 2d24466d5600ea4411e5e847f5be71e5.

[37] M.J. Twiner, S.J. Dixon, C.G. Trick, Extracellular organics from specific cultures of Heterosigma akashiwo (Raphidophyceae) irreversibly alter respiratory activity in mammalian cells, Harmful Algae 3 (2004) 173-182, https://doi.org/10.1016/j.hal. 2003.10.003.

[38] C. Fuentes-Grünewald, E. Garcés, E. Alacid, S. Rossi, J. Camp, Biomass and lipid production of dinoflagellates and raphidophytes in indoor and outdoor photobioreactors, Mar. Biotechnol. 15 (2013) 37-47 http://www.scopus.com/inward/ record.url? eid $=2$-s2.0-84871668893\&partnerID $=40 \& \mathrm{md} 5=$ a5cb16bf5e416822ef830c1b5c718871.

[39] C.M. Bianco, J.J. Stewart, K.R. Miller, C. Fitzgerald, K.J. Coyne, Light intensity impacts the production of biofuel intermediates in Heterosigma akashiwo growing on simulated flue gas containing carbon dioxide and nitric oxide, Bioresour. Technol. 219 (2016) 246-251, https://doi.org/10.1016/j.biortech.2016.07.119.

[40] L. López-Rosales, A. Sánchez-Mirón, A. Contreras-Gómez, F. García-Camacho, F. Battaglia, L. Zhao, E. Molina-Grima, Characterization of bubble column photobioreactors for shear-sensitive microalgae culture, Bioresour. Technol. 275 (2019) 1-9, https://doi.org/10.1016/j.biortech.2018.12.009.

[41] L. López-Rosales, F. García-Camacho, A. Sánchez-Mirón, A. Contreras-Gómez, E. Molina-Grima, Modeling shear-sensitive dinoflagellate microalgae growth in bubble column photobioreactors, Bioresour. Technol. 245 (2017) 250-257, https:// doi.org/10.1016/j.biortech.2017.08.161.

[42] F.R. Nieto, E.J. Cobos, M.Á. Tejada, C. Sánchez-Fernández, R. González-Cano, C.M. Cendán, Tetrodotoxin (TTX) as a therapeutic agent for pain, Mar. Drugs 10 (2012), https://doi.org/10.3390/md10020281.

[43] D.M. Anderson, D.M. Kulis, J.J. Sullivan, S. Hall, C. Lee, Dynamics and physiology of saxitoxin production by the dinoflagellatesAlexandrium spp, Mar. Biol. 104 (1990) 511-524, https://doi.org/10.1007/BF01314358.

[44] A.J. Bourdelais, C.R. Tomas, J. Naar, J. Kubanek, D.G. Baden, New fish-killing alga in coastal Delaware produces neurotoxins, Environ. Health Perspect. 110 (2002) 465-470, https://doi.org/10.1289/ehp.02110465.
[45] C.J. Band-Schmidt, A. Martínez-López, J.J. Bustillos-Guzmán, L. Carreón-Palau, L. Morquecho, N.O. Olguín-Monroy, T. Zenteno-Savín, A. Mendoza-Flores, B. González-Acosta, F.H. Hernández-Sandoval, C. Tomas, Morphology, biochemistry, and growth of raphidophyte strains from the Gulf of California, Hydrobiologia 693 (2012) 81-97, https://doi.org/10.1007/s10750-012-1088-y.

[46] C. Collymore, A. Tolwani, C. Lieggi, S. Rasmussen, Efficacy and safety of 5 anesthetics in adult zebrafish (Danio rerio), J. Am. Assoc. Lab. Anim. Sci. 53 (2014) 198-203 https://www.ncbi.nlm.nih.gov/pubmed/24602548.

[47] T. Martins, A.M. Valentim, N. Pereira, L.M. Antunes, Anaesthesia and analgesia in laboratory adult zebrafish: a question of refinement, Lab. Anim. 50 (2016) 476-488, https://doi.org/10.1177/0023677216670686.

[48] V. Bedell, E. Buglo, D. Marcato, C. Pylatiuk, R. Mikut, J. Stegmaier, W. Scudder, M. Wray, S. Züchner, U. Strähle, R. Peravali, J.E. Dallman, Chapter ten - zebrafish: a pharmacogenetic model for anesthesia, in: R.G. Eckenhoff, I.J.B.T.-M., E. Dmochowski (Eds.), Chem. Biochem. Approaches Study Anesth. Funct. Part a, Academic Press, 2018, pp. 189-209, , https://doi.org/10.1016/bs.mie.2018.02. 004.

[49] J. Lopez-Luna, Q. Al-Jubouri, W. Al-Nuaimy, L.U. Sneddon, Impact of analgesic drugs on the behavioural responses of larval zebrafish to potentially noxious temperatures, Appl. Anim. Behav. Sci. 188 (2017) 97-105, https://doi.org/10.1016/j. applanim.2017.01.002.

[50] J. Lopez-Luna, Q. Al-Jubouri, W. Al-Nuaimy, L.U. Sneddon, Reduction in activity by noxious chemical stimulation is ameliorated by immersion in analgesic drugs in zebrafish, J. Exp. Biol. 220 (2017) 1451 LP-1458, https://doi.org/10.1242/jeb. 146969.

[51] F.J. Sánchez-Vázquez, M.I. Terry, V.O. Felizardo, L.M. Vera, Daily rhythms of toxicity and effectiveness of anesthetics (MS222 and eugenol) in zebrafish (Danio rerio), Chronobiol. Int. 28 (2011) 109-117, https://doi.org/10.3109/07420528. 2010.538105.

[52] M.A. Husen, S. Sharma, Anaesthetic efficacy of MS-222 and AQUI-S ${ }^{\circledR}$ in advanced size fry of rohu, Labeo rohita, (Hamilton-Buchanan), Aquac. Res. 47 (2016) 2496-2505, https://doi.org/10.1111/are.12698. 